\title{
Development of multiple malignancies following long-term glucocorticoid therapy in a patient with leukocytoclastic vasculitis: A case report
}

\author{
LIANGHUA FANG ${ }^{1}$, YUE HU ${ }^{1}$, WEI WANG ${ }^{2}$, SHOUYOU HU ${ }^{1}$, LI ZHANG $^{1}$ and RUIPING WANG ${ }^{1}$ \\ ${ }^{1}$ Department of Oncology, Jiangsu Province Hospital of Traditional Chinese Medicine, Nanjing, Jiangsu 210029, \\ P.R. China; ${ }^{2}$ Department of Hematopathology, MD Anderson Cancer Center, Houston, TX 77030, USA
}

Received December 30, 2015; Accepted April 1, 2016

DOI: $10.3892 / \mathrm{mco} .2016 .854$

\begin{abstract}
Leukocytoclastic vasculitis (LCV) is a neutrophilic inflammation of the blood vessels. LCV may present as a paraneoplastic syndrome occurring before, synchronously with, or after the diagnosis of malignancy. In this study, we report a unique case of multiple malignancies developing simultaneously in a patient with a long history of LCV. The patient was originally diagnosed with LCV and received long-term glucocorticoid treatment. After 11 years of therapy, the patient developed three primary malignancies, including small-cell lung carcinoma, gastric adenocarcinoma and colonic adenocarcinoma. It is likely that LCV was not a paraneoplastic syndrome in this case, but rather an independent process, and the development of multiple cancers is likely associated with the long-term glucocorticoid treatment, which caused imbalance of the immune system. Although the development of cancer during the course of glucocorticoid treatment is very rare, clinicians must be aware of this possible association and immunodysregulation may play a role in this context.
\end{abstract}

\section{Introduction}

Leukocytoclastic vasculitis (LCV) has been associated with several disease processes, such as infections, drugs, allergies, rheumatological and neoplastic diseases (1). In neoplastic processes, LCV may present as a paraneoplastic phenomenon occurring before, synchronously with, or after the diagnosis of malignancy (2). Compared to hematological malignancies, solid tumors are significantly less likely to be associated with LCV (1). The first case of LCV in a patient with a solid tumor was reported in 1968 (3). Since then, more patients suffering from solid tumors and vasculitis have been documented in the

Correspondence to: Dr Ruiping Wang, Department of Oncology, Jiangsu Province Hospital of Traditional Chinese Medicine, 155 Hanzhong Road, Nanjing, Jiangsu 210029, P.R. China

E-mail: 675713760@qq.com

Key words: leukocytoclastic vasculitis, glucocorticoids, malignancy, immune suppression literature (4,5), including patients with lung, colon, gastric and renal cancer. In these previously reported cases, the patients only developed one type of malignancy and the occurrence of multiple malignancies in patients with LCV has not been reported. We herein describe the case of a patient who developed LCV and was treated with glucocorticoids for 11 years. The patient then developed three primary tumors, including small-cell lung carcinoma (SCLC), gastric adenocarcinoma and colonic adenocarcinoma. To the best of our knowledge, this is the first case report of multiple malignancies developing in a patient with a history of LCV in the English medical literature.

\section{Case report}

The patient was a 54-year-old man, initially presenting with rash, purpura, petechiae, bruising and desquamation on the right low limb followed by ulceration in May, 2001 at the age of 43 years. Skin biopsy confirmed the diagnosis of LCV (Fig. 1). The patient was treated with glucocorticoids and initially responded to the steroid therapy very well, achieving complete response. However, the disease later relapsed, and the patient developed dry gangrene of the right foot. Shock therapy with a maximum dose of $500 \mathrm{mg}$ methylprednisolone for 5 days was administered repeatedly. After the disease was controlled, the patient was maintained on methylprednisolone at doses of 80, 60, 40, 20 and $4 \mathrm{mg}$, adjusted according to the disease status over the following 11 years.

In February, 2012, approximately 11 years after the diagnosis of LCV, the patient presented to our hospital complaining of nausea and paroxysmal dull pain in the back and upper abdomen for 3 weeks. The LCV had been stabilized on $4 \mathrm{mg}$ methylprednisolone twice a day. The patient was not a smoker and had no family history of cancer. On physical examination, the patient had a moon facies. No hepatosplenomegaly or lymph node enlargement were observed. There was no jaundice, rash or ulceration. The lower part of the right leg exhibited purple discoloration and desquamation, with marked deformity of the toes, possibly due to the dry gangrene the patient developed due to the deterioration of his LCV (Fig. 2).

The complete blood count revealed a white blood cell count of $10.9 \times 10^{9} / 1$, with an absolute neutrophil count of 


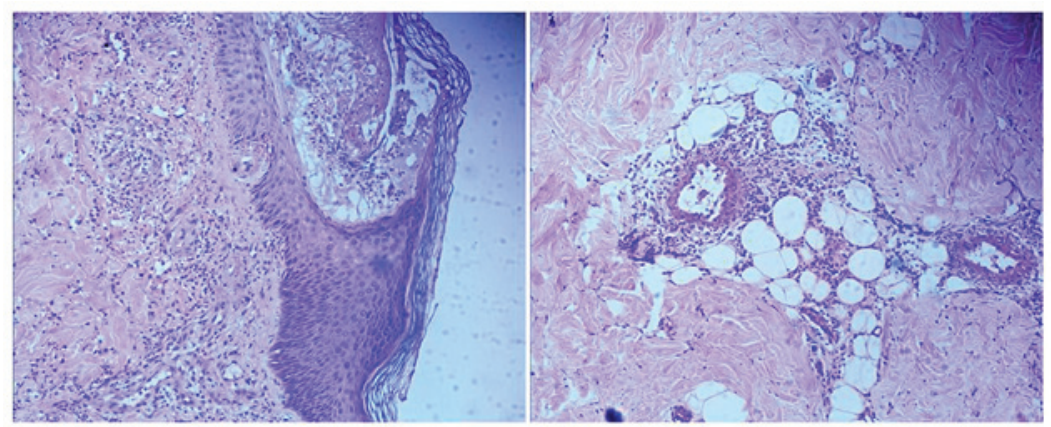

Figure 1. Skin punch biopsy showing the characteristics of leukocytoclastic vasculitis, with perivascular fibrin, neutrophils and leukocytoclasia (nuclear dust) (hematoxylin and eosin staining; magnification, x200).

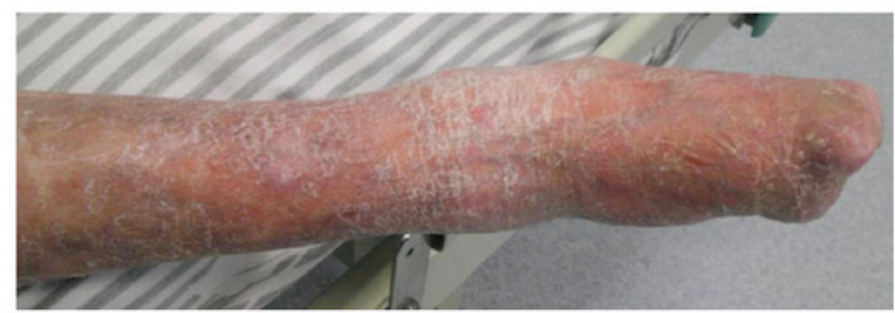

Figure 2. Purple discoloration and desquamation on the lower part of the right leg, with marked deformity of the toes.
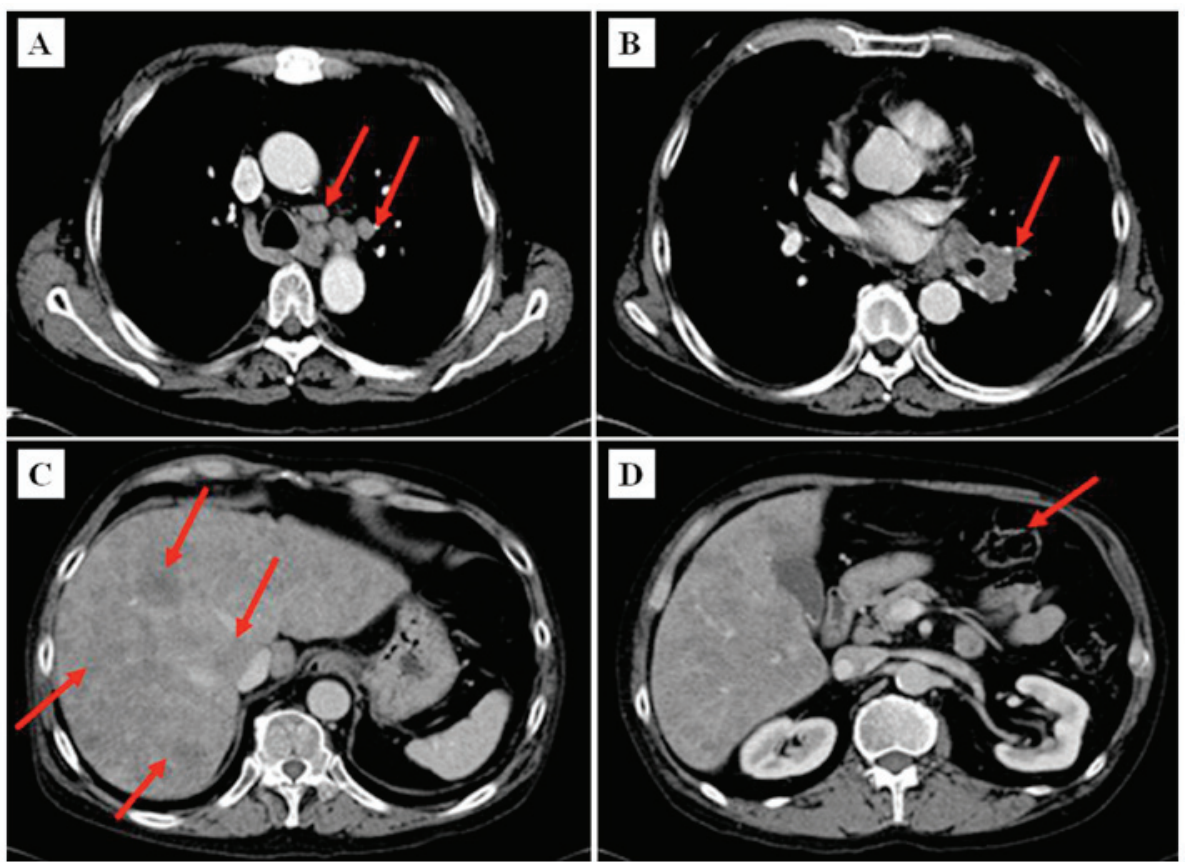

Figure 3. Chest and abdominal computed tomography scans. (A and B) Bulky disease on the left lung hilus, sized $\geq 4.2-5.6 \mathrm{~cm}$, with positive mediastinal lymph nodes, sized $\geq 2-3 \mathrm{~cm}$ (arrows); (C) diffuse metastases in the liver (arrows); (D) incrassation of the gastric wall in the antrum of stomach (arrow).

$8.76 \times 10^{9} / 1$. The percentage of neutrophils and lymphocytes was 80.4 and $8.6 \%$, respectively. The hemoglobin level and platelet count were within normal limits. Routine urine tests showed no positive findings, while the routine stool test was positive for occult blood. Blood chemistry tests revealed an alkaline kinase proteinase level of $341 \mathrm{U} / 1$ (normal, 42-141 U/l), $\gamma$-GT $263 \mathrm{U} / 1$ (normal, 7-32 U/l) and lactate dehydrogenase $624.50 \mathrm{U} / 1$ (normal, 22-29 U/1); others were within the normal range. Tumor marker tests revealed an $\alpha$-fetoprotein level of $3.0 \mathrm{ng} / \mathrm{ml}$ (normal, $<10.9 \mathrm{ng} / \mathrm{ml}$ ), carcinoembryonic antigen $3.63 \mathrm{ng} / \mathrm{ml}$ (normal, $<5 \mathrm{ng} / \mathrm{ml}$ ), ferritin $>1,500 \mathrm{ng} / \mathrm{ml}$ (normal, 11-306.8 $\mathrm{ng} / \mathrm{ml}$ ), carbohydrate antigen (CA) $125>1,000 \mathrm{U} / \mathrm{ml}$ (normal, $<35 \mathrm{U} / \mathrm{ml}$ ), CA153, $78.91 \mathrm{U} / \mathrm{ml}$ (normal, $<31.3 \mathrm{U} / \mathrm{ml}$ ) and CA199, $112.28 \mathrm{U} / \mathrm{ml}$ (normal, $<37 \mathrm{U} / \mathrm{ml}$ ). The results of the cellular immunity tests were as follows: CD3, 37\% (normal, 50-84\%); CD19, 4\% (normal, 5-18\%), CD4, 20\% (normal, 27-51\%), CD8, 50\% (normal, 15-44\%); and CD4/CD8 ratio, 0.4 (normal, 0.71-2.78). 


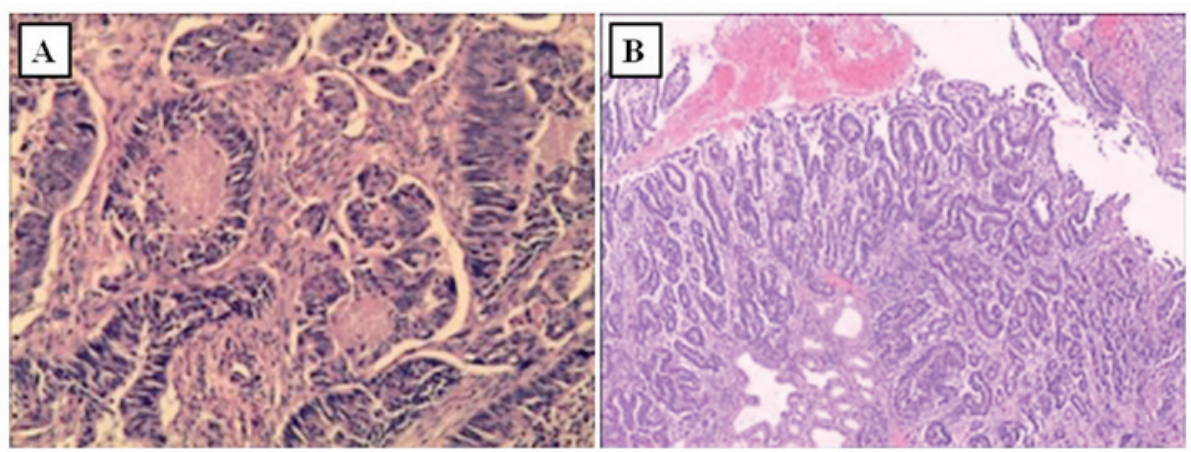

Figure 4. Hematoxylin and eosin staining of (A) colon biopsy, showing ulcerated type tubular adenocarcinoma with central necrosis, characteristic of colonic adenocarcinoma (magnification, x200) and (B) stomach biopsy, showing moderately differentiated adenocarcinoma (magnification, x100).
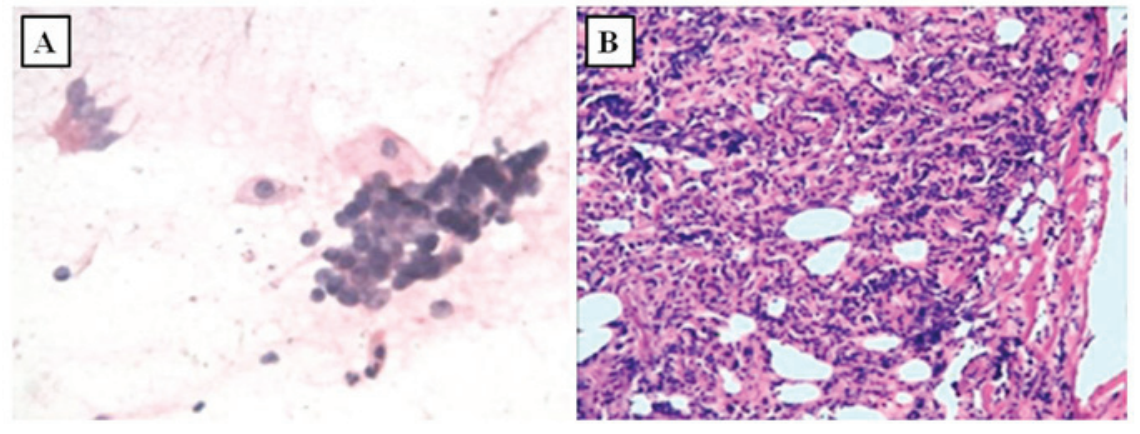

Figure 5. (A) Bronchial brushing smear. Clusters of malignant cells were identified (magnification, x200). (B) Biopsy showing small-cell lung carcinoma (magnification, x100). Hematoxylin and eosin staining was used (panels A and B).

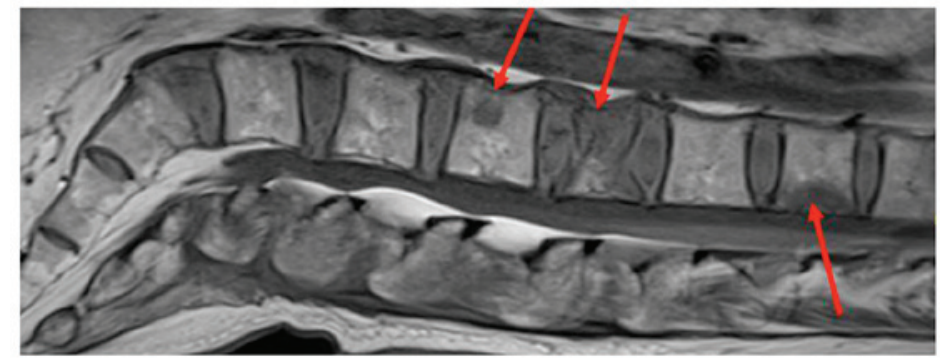

Figure 6. Vertebral magnetic resonance imaging showing multiple metastases to the spine, ranging from the twelfth thoracic to the second sacral vertebra (arrows), with a compression fracture of the second lumbar vertebra.

A CT scan of the chest and abdomen was performed and revealed bulky disease at the left lung hilus sized $\leq 4.2-5.6 \mathrm{~cm}$, enlarged mediastinal lymph nodes sized $\leq 2-3 \mathrm{~cm}$ (Fig. 3A-B) and diffuse liver metastases (Fig. 3C). In addition, minor incrassation of the gastric wall in the antrum of stomach was present (Fig. 3D).

Colonoscopy and esophogastroduodenoscopy with concurrent biopsies were performed. Colonoscopy revealed an ulcerated lesion at the sigmoid region, measuring $\sim 1 \mathrm{x} 1.5 \mathrm{~cm}$. Biopsy of the lesion (Fig. 4A) showed ulcerated type tubular adenocarcinoma. Esophogastroduodenoscopy revealed antral ulceration; the examination for Helicobacter pylori (H.pylori) was negative and the biopsy showed gastric adenocarcinoma, moderately differentiated (Fig. 4B). To confirm the pathology of the hilar mass in the left lung, bronchoscopy was performed and revealed left hilar enlargement. A bronchial brushing smear revealed clusters of malignant cells (Fig. 5A). SCLC was suspected and the diagnosis was confirmed by biopsy (Fig. 5B).

Due to back pain, vertebral magnetic resonance imaging was performed, which revealed multiple metastases to the spine, from the twelfth thoracic to the second sacral vertebra. A vertebral compression fracture of the second lumbar vertebra was also identified (Fig. 6).

In summary, the patient was diagnosed with three separate maligancies: Gastric adenocarcinoma, colonic adenocarcinoma and SCLC, with stage IV metastatic disease. The patient received palliative chemotherapy, which included paclitaxel $120 \mathrm{mg}$ (days 1 and 8), etoposide $100 \mathrm{mg}$ (days 1-5) and cisplatin $20 \mathrm{mg}$ (days 1-5). During the course of chemotherapy, grade 4 bone marrow suppression ensued, complicated by pulmonary Pseudomonas aeruginosa infection. The patient was started on antibiotics and chemotherapy was terminated. However, his condition deteriorated rapidly, with pronounced 
abdominal distention, continuously declining urine volume, liver enlargement and ascites. During this period there was no progression of LCV and no new rash, purpura, petechia or ulceration were identified. The patient succumbed 3 months after the diagnosis of the cancers.

\section{Discussion}

LCV is a neutrophilic inflammation of the blood vessels. As a paraneoplastic syndrome, it may occur prior to the diagnosis of the primary tumor. The exact association between LCV and malignancy has not been fully elucidated. Various mechanisms have been proposed for tumor-associated LCV, including antigen-antibody complexes that form in response to tumor antigens and are deposited in vessel walls, resulting in inflammation (6). Malignant neoplasms may also increase blood viscosity, causing potential endothelial damage and increasing contact time for immune complex deposition (7). $\mathrm{LCV}$, when presenting as a paraneoplastic syndrome, is usually refrractory to corticosteroid and immunosuppressant treatment, whereas its symptoms improve with effective treatment of the underlying malignancy. Moreover, recurrence of LCV often occurs with the progression or metastasis of the malignancy (8). In our case, LCV occurred 11 years prior to the development of the malignancies. The patient responded to methylprednisolone therapy and there was no deterioration of LCV when the malignancies were diagnosed. Thus, we hypothesize that LCV was not a paranoeplastic syndrome, but rather an independent condition in our patient. However, it is likely that the immunosuppression triggered by the long-term treatment of LCV with glucocorticoids contributed to the development of multiple malignancies.

It is well known that glucocorticoid administration inhibits the immune system, including induction of apoptosis in T lymphocytes (9) and inhibition of natural killer cell activity (10). These have been associated with increased susceptibility to tumor development (11). Furthermore, glucocorticoids appear to contribute to the shift of $\mathrm{T}$ helper cells from the Th1 to the Th2 phenotype (9), which facilitates cancer escape from host immune surveillance (12). It was previously demonstrated that patients with immunological disorders treated with glucocorticoids displayed a high incidence of secondary cancers (13). In our case, the patient had a history of LCV and received long-term glucocorticoid treatment, with the maximum dose reaching $500 \mathrm{mg} /$ day of methylprednisolone. The disruption of the immune system by steroid treatment is likely the cause of cancer development. Other cancer risk factors were absent in this patient, as he was not a smoker and there was no $H$. pylori infection identified in the gastric biopsy.

To the best of our knowledge, this is the first reported case of multiple malignancies developing after long-term steroid treatment in a patient with LCV. LCV in this case was not a paraneoplastic syndrome, and the development of the malignancies is likely associated with immune dysregulation by the steroid treatment. This case report suggests that clinicians should be aware of the possible association of long-term use of glucocorticoids with the development of secondary malignancies.

\section{Acknowledgements}

The present study was supported by grants from the Natural Science Youth Foundation of Jiangsu Province (BK20141034) and the Project of Administration of Traditional Chinese Medicine Research of Jiangsu Province (LZ13051), the 'Top Talented Personnel in Six Profession' grant in Jiangsu Province (2011-WS-049) and a grant from the Jiangsu Province Hospital of Traditional Chinese Medicine (2013, Y1008).

\section{References}

1. Langford CA: Vasculitis. J Allergy Clin Immunol 125 (2 Suppl 2): S216-S225, 2010.

2. Fain O, Hamidou M, Cacoub P, Godeau B, Wechsler B, Pariès J, Stirnemann J, Morin AS, Gatfosse M, Hanslik T, et al: Vasculitides associated with malignancies: Analysis of sixty patients. Arthritis Rheum 57: 1473-1480, 2007.

3. Torvik A and Berntzen AE: Necrotizing vasculitis without visceral involvement: Postmortem examination of three cases with affection of skeletal muscles and peripheral nerves. Acta Med Scand 184: 69-77, 1968.

4. Podjasek JO, Wetter DA, Pittelkow MR and Wada DA: Cutaneous small-vessel vasculitis associated with solid organ malignancies: The Mayo Clinic experience, 1996 to 2009. J Am Acad Dermatol 66: e55-e65, 2012.

5. Kathula SK, Thomas DE, Anstadt MP and Khan AU: Paraneoplastic cutaneous leukocytoclastic vasculitis and iron deficiency anemia as the presenting features of squamous cell lung carcinoma. J Clin Oncol 29: e83-e85, 2011.

6. Greer JM, Longley S, Edwards NL, Elfenbein GJ and Panush RS: Vasculitis associated with malignancy: Experience with 13 patients and literature review. Medicine (Baltimore) 67: 220-230, 1988.

7. Magro CM and Crowson AN: A clinical and histologic study of 37cases of immunoglobulin A-associated vasculitis. Am J Dermatopathol 21: 234-240, 1999.

8. Sánchez-Guerrero J, Gutiérrez-Ureña S, Vidaller A, Reyes E, Iglesias A and Alarcón-Segovia D: Vasculitis as a paraneoplastic syndrome: Report of 11 cases and review of the literature. J Rheumatol 17: 1458-1462, 1990.

9. Tuckermann JP, Kleiman A, McPherson KG and Reichardt HM: Molecular mechanisms of glucocorticoids in the control of inflammation and lymphocyte apoptosis. Crit Rev Clin Lab Sci 42: 71-104, 2005.

10. Oshimi K, Gonda N, Sumiya M and Kano S: Effects of corticosteroids on natural killer activity in systemic lupus ery thematosus. Clin Exp Immunol 40: 83-88, 1980.

11. Riley V: Psychoneuroendocrine influences on immunecompetence and neoplasia. Science 212: 1100-1109, 1981.

12. Asselin-Paturel C, Echchakir H, Carayol G, Gay F, Opolon P, Grunenwald D, Chouaib S and Mami-Chouaib F: Quantitative analysis of Th1, Th2 and TGF-beta1 cytokine expression in tumor, TIL and PBL of non-small cell lung cancer patients. Int J Cancer 77: 7-12, 1998.

13. Klumb EM, Araújo ML Jr, Jesus GR, Santos DB, Oliveira AV, Albuquerque EM and Macedo JM: Is higher prevalence of cervical intraepithelial neoplasia in women with lupus due to immunosuppression? J Clin Rheumatol 16: 153-157, 2010. 